\title{
EFEITO DO PROCESSAMENTO E ESTOCAGEM NA CONCENTRAÇÃO DE SUBSTÂNCIAS BIOATIVAS EM ALIMENTOS
}

CINTIA ALESSANDRA MATIUCCI PEREIRA*

O presente trabalho teve como objetivo reunir dados da literatura sobre os efeitos do processamento e da estocagem na concentração de substâncias bioativas em alimentos. Foram abordados os vegetais Brassica, as substâncias fenólicas presentes em frutas, o licopeno em tomates, os vegetais minimamente processados e os alimentos orgânicos. Pode ser observado que ainda são escassos estudos que relacionem a influência do processamento e da estocagem na diminuição ou aumento das substâncias bioativas. Os dados disponíveis na literatura demonstram que há problemas técnicos a serem resolvidos, como a disponibilidade de métodos analíticos para quantificação dessas substâncias e o entendimento, ainda limitado, sobre os efeitos na sua estabilidade.

* Professora, Doutora em Ciências, Centro Universitário Central Paulista (UNICEP), São Carlos, SP (e-mail: cmatiucci@gmail.com). 


\section{INTRODUÇÃO}

Embora remonte às origens da civilização, a relação entre alimentação e saúde vem se estreitando cada vez mais. Dietas ricas em gordura, sal e açúcar e pobres em carboidratos complexos, vitaminas e minerais, aliadas ao estilo de vida mais sedentário, são responsáveis pelo aumento de doenças como obesidade, diabetes, problemas cardiovasculares, hipertensão, osteoporose e câncer. Há muito tempo acredita-se que o consumo de frutas e hortaliças auxilia na prevenção dessas doenças. Além da nutrição básica, seus compostos funcionais beneficiam uma ou mais funções orgânicas, contribuindo para melhorar o estado de saúde e bem-estar e/ou reduzir o risco de doenças crônicodegenerativas (ADA, 2004; STRINGHETA et al., 2007).

As primeiras pesquisas sobre alimentos funcionais tiveram início há 20 anos. O mercado global de alimentos e bebidas funcionais e fortificados movimentou cerca de 60 bilhões de dólares em 2002; 80 bilhões em 2005 e movimentará 110 bilhões em 2010 (HARDY, 2000; SWADLING, 2001; MOTTA, 2006). Os fatores que impulsionam o mercado global de saúde e bem-estar podem ser associados à demanda, ao abastecimento e aos governos. Segundo BURDOCK, CARABIN e GRIFFTHS (2006), aproximadamente 158 milhões de americanos gastaram, em 2004, cerca de 20,2 bilhões de dólares em alimentos funcionais, nutracêuticos e suplementos dietéticos. Tal valor representa mais que o dobro do montante gasto em 1994. Uma das razões para o aumento do consumo desses produtos é tentar suprir a deficiência de atividade física e de hábitos de alimentação saudáveis, próprios da vida urbana convencional. Outras razões envolvem o alto custo dos medicamentos, a tendência de uso de produtos mais naturais, ou simplesmente a adoção de medidas preventivas como, por exemplo, o consumo de fibras. Trata-se, portanto, de segmento de grande interesse às indústrias alimentícias, que têm buscado explorar a relação entre o consumo de determinados ingredientes com a redução de fatores de risco para doenças específicas, principalmente as crônico degenerativas, ou a melhoria da performance física ou mental (GRIZARD, DALLE e BARTHOMEUF, 2001; IZZO e NINESS, 2001).

BATTAGLIA (2006) dividiu em duas, as fases do mercado de alimentos funcionais. A primeira ocorreu na década de noventa com a adoção desse conceito (estimulado pelas inovações do mercado japonês dos anos 80), de testes de mercado e de sucessos como, os probióticos, margarinas para redução do colesterol, barras de cereais e outros. A segunda fase, iniciadas em 2000 , caracterizou-se pela consolidação dos líderes da indústria de alimentos no mercado nutricional, a diversificação de produtos para os mais variados temas de saúde e o reposicionamento de alimentos convencionais à luz da ciência da nutrição, além do crescente foco das empresas na percepção do consumidor. Também se observa a crescente confiança na ciência para encontrar resposta à questão "dieta e saúde" mediante novos ingredientes funcionais como flavonóides, isoflavonas, organossulfurados, fitoesterois, entre outros, chamadas de substâncias bioativas (NAMIKI, 1990).

A efetividade dos compostos bioativos em produtos alimentícios pode ser afetada pelo processamento (DEKKER et al., 1999; KLOPOTEK, OTTO e BOHM, 2005). Assim, o objetivo desta revisão foi relatar estudos sobre a influência do processamento e da estocagem na concentração de substâncias bioativas em alimentos.

\section{EFEITO DO PROCESSAMENTO NAS SUBSTÂNCIAS BIOATIVAS}

O princípio básico do processamento de alimentos é retardar a atividade microbiana e alterações químicas que podem afetar sua qualidade. Entretanto, devido à severidade dos processos, o alimento geralmente sofre mudanças extensivas e como resultado suas características de qualidade podem ser modificadas. Algumas alterações são desejadas (como, por exemplo, a inativação de fatores antinutricionais pelo calor, o amaciamento de tecidos e a criação de aromas) ou indesejáveis (como perda de vitaminas ou de cor, mudanças na textura e produção de substâncias que interferem no aroma) e podem ser inaceitáveis aos consumidores (BRECHT et al., 2008). 
Os vegetais frequentemente passam por várias formas de processamento para que fiquem mais adequados ao consumo, ou mais duráveis à longa estocagem. O processamento térmico úmido pode afetar os fitoquímicos pela quebra térmica, sendo a integridade da estrutura celular perdida pela migração dos componentes, permitindo perdas por escoamento ou por ação enzimática, além da atuação de fatores não enzimáticos como luz e oxigênio (VOLDEN et al., 2008). Entretanto, quando otimizado, o tratamento térmico irá rapidamente inativar enzimas, prevenir o acesso do oxigênio e minimizar perdas.

Os vegetais da família Brassicaceae (Cruciferae), brócolis, couve de bruxelas, repolho e mostarda servem como exemplo. As propriedades das Brassicas incluem a ação direta ou indireta como antioxidantes, controle da apoptose e ciclo celular (MORENO et al., 2006). Esses vegetais contêm glucosinolatos, produtos de quebra metabólica que são potentes moduladores das enzimas xenobióticas que protegem o DNA do perigo. A alta ingestão de crucíferas está associada com o risco reduzido de câncer, particularmente de pulmão e do trato gastrointestinal. A literatura epidemiológica reforça a hipótese de que alta ingestão de Brassicas reduz o risco do câncer de próstata (KRISTAL e LAMPE, 2002).

A ação quimopreventiva das Brassicas está relacionada ao nível de glucosinolatos, sua conversão a isotiocianatos e biodisponibilidade dos metabólitos (SONG e THORNALLEY, 2007). Os glucosinolatos são quimicamente estáveis até terem contato com a enzima mirosinase, a qual está estocada e compartamentalizada no tecido (KELLY, BONES e ROSSITER, 1998). A mirosinase é liberada quando o vegetal sofre ação de insetos ou ao ser congelado, descongelado, cortado e picado (SONG e THORNALLEY, 2007). Assim, a estocagem e o processamento podem afetar o teor dessas substâncias.

O processamento culinário exerce influências complexas no teor de nutrientes e não nutrientes. O cozimento a altas temperaturas desnatura a mirosinase no material vegetal, resultando em conversão mais baixa de glucosinolatos a isotiocianatos quando mastigados. Os glucosinolatos dos vegetais consumidos são degradados a isotiocianatos por ação da mirosinase das bactérias intestinais (SHAPIRO et al., 2001). A estocagem a $-8,5^{\circ} \mathrm{C}$ pode causar significante perda de glucosinolatos devido à quebra das células da planta durante o congelamento e acessabilidade da mirosinase a glucosinolatos com subsequente conversão enzimática a isotiocianatos durante o descongelamento (SONG e THORNALLEY, 2007).

Geralmente, os vegetais são estocados entre 4 e $8^{\circ} \mathrm{C}$ no refrigerador ou à temperatura ambiente por até uma semana. Ao se cortar o vegetal, $75 \%$ dos glucosinolatos são perdidos durante as próximas 6 horas em temperatura ambiente, o que poderia diminuir, por exemplo, a qualidade dos vegetais minimamente processados. A redução no teor de glucosinolatos ocorre pela exposição à mirosinase (SONG e THORNALLEY, 2007).

SONG e THORNALLEY (2007) demonstraram diminuição significante de glucosinolatos em vegetais cozidos em água. Entretanto, o cozimento a vapor, em micro-ondas e fritura exercem pouco efeito no teor total de glucosinolatos. ZHANG e HAMAUZU (2004) cozinharam brócolis por 5 minutos usando a fração de vegetal:água de 1:20, e encontraram reduções significativas de 72\%, 66\%, e 65\% dos fenólicos totais, L-ácido ascórbico e atividade antioxidante, respectivamente. HALVORSEN et al. (2002) relataram aumentos na atividade antioxidante de muitos vegetais depois de cozidos em microondas, no vapor e em água. WENNBERG et al. (2006) investigaram os níveis de glicosinolatos em dois cultivares de repolho branco após branqueamento e encontraram redução de 50 e 74\%.

O processamento industrial pode afetar os níveis de glucosinolatos e dos produtos de quebra dos vegetais Brassica de modo similar ao processamento culinário doméstico. Todas as operações pós-colheita disparam complexas reações que modificam seu o teor de glucosinolatos (GOODRICH, ANDERSON e STOEWSAND, 1989; DAXENBICHLER et al., 1991; WATHELET et al., 1996; MITHEN et al., 2000). 
Em muitos estudos sobre o efeito do processamento térmico em Brassicas, somente um tipo de substância bioativa tem sido investigado. Assim o comportamento e as relações entre os vários constituintes em condições experimentais continuam desconhecidos (VOLDEN et al., 2008). No brócolis, por exemplo, o foco se concentra no composto bioativo sulforafano (KRISTAL e LAMPE, 2002; FINLEY, 2005; JACKSON e SINGLETARY, 2004), embora apresente flavonóides e MANACH et al. (2004) já tenham encontrado alta concentração de quercetina nesse vegetal.

\subsection{EFEITO DO PROCESSAMENTO EM SUBSTÂNCIAS FENÓLICAS}

Quando se discute a presença das substâncias bioativas em alimentos, os flavonóides são a classe mais descrita porque representam aproximadamente 2/3 dos fenólicos dietéticos (ROBBINS, 2003). Os ácidos fenólicos se comportam como antioxidantes, devido à reatividade da porção fenol (substituinte hidroxil no anel aromático). Há muitos mecanismos, mas acredita-se que o principal seja como sequestrante de radicais via doação do átomo de hidrogênio (ROBBINS, 2003).

Sucos de frutas, chás e vinhos são importantes fontes de fenólicos na dieta humana e a redução desses compostos, relacionada aos processamentos industriais, pode diminuir sua capacidade antioxidante (DEKKER et al., 1999; GIL-IZQUIERDO, GIL e FERRERES, 2002; BALASUNDRAM, SUNDRAM e SAMMAN, 2006). O processamento afeta o conteúdo, a atividade e a biodisponibilidade dos componentes bioativos dos alimentos (NICOLI, ANESE e PARPINEL, 1999; CARVALHO et al., 2000; MAIA et al., 2007).

A vitamina $C$ representa o componente mais avaliado em alimentos e sua perda varia de acordo com o processo e equipamentos utilizados (ACHINEWHU e HARD, 1994; GIMENEZ et al., 2002; MATSUURA et al., 2002; GAHLER, OTTO e BOHM, 2003; YAMASHITA et al., 2003), sendo que matérias-prima fontes dessa vitamina ainda retêm alto conteúdo após o processamento (SEMENSATO, 1997). A degradação do ácido ascórbico pode ocorrer em condições aeróbicas ou anaeróbicas, ambas levando à formação de pigmentos escuros e também pela ação da luz. Sua estabilidade aumenta com a diminuição da temperatura (PERERA e BALDWIN, 2001).

Em suco de uva industrializado, catequinas, miricetina e kaempferol são mais suscetíveis ao processamento do que a quercetina (ARTS, VAN DE PUTTE e HOLLMAN, 2000; HAKKINEN et al., 2000).

A maior perda de fenólicos em suco de maçã ocorre na prensagem (DEKKER et al., 1999). A concentração de ácido clorogênico em suco industrializado representa somente $50 \%$ da encontrada no suco fresco (VAN DER SLUIS et al., 2002). Há relatos que a utilização de micro-ondas aumenta o conteúdo de substâncias fenólicas em suco de maçã (valores acima de 65 mg/100 mL) (GERARD e ROBERTS, 2004). Em baixas temperaturas de estocagem, o teor de fenólicos se mantém estável (DEKKER et al., 1999; VAN DER SLUIS, DEKKER e VAN BOEKEL, 2005).

Os morangos, consumidos principalmente como fruta fresca, podem ser utilizados para a elaboração de sucos, néctares, purês, suco concentrado e geléia. As etapas do processamento em comum são: concentração do suco de fruta, estocagem em tanques, rediluição, produção da geléia por aquecimento a vácuo, acondicionamento nas embalagens, fechamento sob vácuo e resfriamento (KLOPOTEK, OTTO e BOHM, 2005). KLOPOTEK, OTTO e BOHM (2005) analisaram suco de morango e observaram diminuição dos compostos fenólicos com o avanço do tempo de processamento e do estágio de produção. As principais perdas das substâncias fenólicas ocorreram na pasteurização do suco (-27\%), na produção do néctar (-14\%), na trituração da fruta (-15\%) e na prensagem da fruta (-29\%), sendo menor na etapa de filtração do suco.

GIL-IZQUIERDO, GIL e FERRERES (2002) investigaram os efeitos do processamento em suco de laranja em escala industrial (pasteurização, concentração e congelamento) e dois métodos de extração do suco, com relação ao teor de fenólicos, vitamina $C$ e atividade antioxidante. $O$ método de extração constitui o ponto crítico na elaboração de suco de laranja porque são encontradas flavanonas 
em concentração muito mais alta no albedo e na casca que na parte comestível. Na prática, a extração doméstica rende sucos mais ricos em compostos fenólicos solúveis. Por outro lado, a extração comercial é mais vigorosa e produz sucos com alta concentração de compostos fenólicos e vitamina C. A pasteurização não modificou o teor nutricional e do antioxidante nos sucos estudados, mas degradou os fenólicos derivados do ácido cafeico, 2-vicenina e narirutina. O congelamento provocou dramática diminuição no teor de fenólicos, e a concentração causou precipitação no suco não clarificado.

Em frutas do gênero Citrus, os flavonóides estão presentes principalmente na forma glicosilada. As agliconas ocorrem menos frequentemente devido à sua natureza lipofílica e baixa solubilidade em água. A presença de grande número de flavonóides em Citrus resulta das muitas combinações que são possíveis entre agliconas polihidroxiladas e mono e dissacarídeos. Polimetoxiflavonas, presentes na casca de Citrus, são encontradas em maior concentração nos sucos industrializados, pois o processo permite que o suco seja "contaminado" com constituintes da casca (GATTUSO et al., 2007). Os sucos caseiros contêm traços desses compostos, os quais dificilmente podem ser detectados.

O processo de clarificação na indústria de sucos assume fundamental importância para remover a turbidez e prevenir o eventual desenvolvimento de precipitados durante a estocagem ou depois da reconstituição do suco concentrado. A presença de fragmentos de células e pequenas moléculas de pectina insolúveis são as responsáveis pela turbidez imediata em sucos frescos, cujo desenvolvimento pode ser resultado das interações entre polissacarídeos, açúcares, íons metálicos e proteínas ou devido à polimerização oxidativa entre polifenóis e proteínas (SIEBERT, CARRASCO e LYNN, 1996; SIEBERT, TROUKHANOVA e LYNN, 1996; BEVERIDGE, 1997; SIEBERT, 1999). Entre os procedimentos mais empregados para remover a turbidez destaca-se o uso de gelatina e sílica, que podem causar redução substancial de compostos fenólicos (LANDBO et al., 2006).

Os resíduos agroindustriais são boas fontes de compostos fenólicos e dessa forma tem sido explorados como fonte de antioxidantes naturais (SCHIEBER, STINTZING e CARLE, 2001; BALASUNDRAM, SUNDRAM e SAMMAN, 2006). A indústria de Citrus, por exemplo, produz grande quantidade de resíduo de casca e semente que pode representar $50 \%$ do peso total da fruta. Esses resíduos contêm até 15\% a mais de fenólicos totais que a parte comestível (BALASUNDRAM, SUNDRAM e SAMMAN, 2006).

O licopeno, um dos carotenóides mais estudados, permanece relativamente estável durante procedimentos típicos de processamento, exceto em condições extremas (como altas temperaturas ou tempo de aquecimento muito longo). A isomerização trans e cis pode ocorrer durante o processamento, especialmente na presença de gordura, e a reisomerização durante o tempo de estocagem. Luz e oxigênio podem acelerar as alterações durante o processamento e a estocagem, devendo ser evitados. Autoxidação também pode ocorrer e induzir a formação de aroma indesejáveis (XIANQUAN et al., 2005).

Ao contrário do que se imaginava, o cozimento de alimentos ricos em licopeno resulta em redução mínima dessa substância. A ação do calor no alimento, somada à ingestão de gorduras aumenta a biodisponibilidade e a absorção do licopeno. Ao cozinhar o suco de tomate com $1 \%$ de óleo de milho aumenta-se de duas a três vezes a concentração do licopeno sérico (STAHL e SIES, 1992). Estudos mostraram que a ação do calor resulta em maior concentração de isômeros cis, sugerindo que essa configuração é mais facilmente absorvida.

GAHLER, OTTO e BOHM (2003) investigaram o modo como os tratamentos industriais e caseiros podem afetar compostos fenólicos em molhos e sopas de tomates, cuja distribuição também varia nas diferentes partes do fruto. A parte externa do tomate apresentou valores maiores de fenólicos quando comparada à parte interna, sendo encontrados $31 \mathrm{mg} / 100 \mathrm{~g}$ de fenólicos totais nas sementes e $27 \mathrm{mg} / 100 \mathrm{~g}$ na parte externa. Não foram verificadas diferenças significativas no teor de fenólicos durante o processamento do suco de tomate. Os tratamentos de 15, 30 e 45 minutos a 180, 200 e $220^{\circ} \mathrm{C}$ aumentaram o teor de fenólicos totais, possivelmente devido sua liberação da matriz.

TOOR e SAVAGE (2006a) investigaram a influência da estocagem (7, 15 e 25드. por 10 dias) nos compostos bioativos de tomate. Os fenólicos solúveis e o ácido ascórbico revelaram pequena 
elevação durante a estocagem, independentemente da temperatura empregada. O teor de licopeno em tomates armazenados a 15 e $25^{\circ} \mathrm{C}$ foi duas vezes superior ao estocado a $7^{\circ} \mathrm{C}$. A atividade antioxidante aumentou de 17 a $27 \%$ durante a estocagem, provavelmente pelo efeito sinergístico entre os compostos fenólicos e o ácido ascórbico.

Em tomates, o cozimento e a fritura resultaram em significante redução do ácido ascórbico, fenólicos totais e licopeno (SAHLIN, SAVAGE e LISTER, 2004). Após o processo de secagem ocorre elevação do teor de flavonóides, fenólicos e licopeno, acarretando aumento da atividade antioxidante (CHANG et al., 2006). A secagem pode acelerar a liberação dos fenólicos a partir da quebra dos constituintes celulares. Embora o rompimento das paredes celulares possa liberar enzimas oxidativas e hidrolíticas que poderiam destruir os antioxidantes nas frutas, a alta temperatura do processo de secagem com ar quente desativa as enzimas e permite o aumento dos fenólicos. BOVY et al. (2002) relataram que os tomates contêm alguns tipos de flavonóides na pele, principalmente naringenina, chalcona e rutina. Assim, o aumento na quantia de flavonóides pode ser explicado pelas reações bioquímicas que ocorrem na pele do tomate durante a secagem. Entretanto, TOOR e SAVAGE (2006b) verificaram redução entre 10 a 15\% dos flavonóides como resultado da semi-secagem em baixas temperaturas $\left(42^{\circ} \mathrm{C}\right)$. Essa perda pode decorrer da ação das enzimas oxidativas como polifenoloxidases e peroxidases, que não são inativadas nessa faixa de temperatura. Por outro lado, a perda de ácido ascórbico depende da temperatura de secagem utilizada. Tomates secos a $80^{\circ} \mathrm{C}$ contém $10 \%$ de ácido ascórbico, enquanto os secos a $110^{\circ} \mathrm{C}$ não apresentam mais essa substância (TOOR e SAVAGE, 2006b).

\subsection{VEGETAIS MINIMAMENTE PROCESSADOS E ORGÂNICOS}

O processamento mínimo pode ser definido como a combinação de procedimentos, como lavagem, classificação, corte, descascamento e fatiamento que não afetam a qualidade de frescor do alimento (ODRIOZOLA-SERRANO, SOLIVA-FORTUNY e MARTIN-BELLOSO, 2008). Consequentemente, os vegetais e frutas minimamente processados são alimentos frescos, altamente nutritivos, práticos e de qualidade. Entretanto, como resultado do processo, vários fenômenos fisiológicos como mudanças bioquímicas e contaminação microbiológica podem ocorrer, resultando na perda de cor, textura e aroma do alimento (ODRIOZOLA-SERRANO, SOLIVA-FORTUNY e MARTIN-BELLOSO, 2008). Pouca informação está disponível na literatura sobre a influência do processamento mínimo no conteúdo de compostos bioativos nesses alimentos.

ODRIOZOLA-SERRANO, SOLIVA-FORTUNY e MARTIN-BELLOSO (2008) investigaram o teor de licopeno, de vitamina $\mathrm{C}$ e de compostos fenólicos, além da atividade antioxidante em diferentes cultivares de tomates minimamente processados e mantidos a $4^{\circ} \mathrm{C}$ pós-colheita. Não encontraram diferença significativa com relação aos vários cultivares e aos parâmetros estudados, exceto para o teor de fenólicos totais que diminuiu de maneira significativa a partir do $15^{\circ}$ dia de estocagem.

O sistema agrícola pode ser determinante na qualidade de vegetais e frutos produzidos. Os alimentos orgânicos vem ganhando cada vez mais espaço nos mercados brasileiros. Estudo sobre os fatores que afetam a escolha de alimentos vegetais pelos consumidores mostrou que as ideologias pessoais aumentaram a porcentagem de venda de produtos orgânicos, apesar do seu alto custo (BARRETT, WEAKLEY e WATNIK, 2007).

Há evidência científica que os alimentos orgânicos contêm maiores teores de minerais e vitaminas, melhor sabor e maior atividade antioxidante (BARRETT, WEAKLEY e WATNIK, 2007). Conforme o INSTITUT OF FOOD TECHNOLOGISTS (2006) ainda é prematuro afirmar se o sistema orgânico é superior ao convencional com relação à composição nutricional dos alimentos (BARRETT, WEAKLEY e WATNIK, 2007). Algumas substâncias bioativas são estimuladas em sistemas orgânicos de produção. O resveratrol, encontrado em sucos de uva e amora, tem sua produção estimulada por ataque de fungos, estresse (irradiação, calor e toxinas) e radiação ultravioleta. O suco de uva orgânico 
apresenta maior concentração de resveratrol, pois a planta desenvolve mais a autodefesa sem uso de agrotóxicos (SOUTO, 2007).

LESTER, MANTHEY e BUSLIG (2007) compararam os fenólicos (flavonóides e furanocumarinas) do suco de grapefruit orgânico e convencional por cromatografia a líquido de alta eficiência com detector de arranjo de diodos acoplada a espectrômetro de massas (HPLC/DAD/MS) e encontraram maior teor em sucos orgânicos. Além do sistema de produção houve influência do tempo de colheita, sendo as furanocumarinas as mais influenciadas. Esses resultados são similares aos encontrados por LOMBARDIBOCCIA et al. (2004) estudando ameixas orgânicas e convencionais.

LESTER, MANTHEY e BUSLIG (2007) apontam a necessidade de mais estudos que demonstrem as diferenças entre produtos orgânicos e convencionais. Poucos são os trabalhos que relatam a qualidade de frutas e sucos enfocando os compostos bioativos.

\section{CONCLUSÃO}

Em consequência da aceitação e necessidade dos alimentos funcionais, a indústria alimentícia passa por transformações. No entanto, a influência do processamento e da estocagem na concentração das substâncias bioativas ainda precisa ser mais estudada. Diversos cultivares e várias condições de crescimento, além das diferenças na execução dos processos dificultam as interpretações e comparações entre os estudos. A dificuldade para quantificar essas substâncias está associada a vários problemas técnicos que ainda não foram resolvidos: (i) a disponibilidade de métodos analíticos validados para a quantificação; $\mathrm{e}$ (ii) o entendimento limitado dos efeitos da estocagem e processamentos na estabilidade das substâncias.

\section{ABSTRACT}

\section{EFFECTS OF PROCESSING AND STORAGE IN THE CONCENTRATION OF BIOACTIVE SUBSTANCES IN FOOD}

This study gathered data from the literature about the effects of processing and storage in the concentration of bioactive substances in food. The review main focuses were Brassica vegetables, phenolic substances present in fruits, lycopene in tomatoes, vegetables minimally processed and organic foods. It was observed that there are few studies correlating the influence of processing and storage at the decrease or increase of bioactive substances. The available literature shows that there are technical problems to be solved, as the availability of analytical methods for quantification, and limited knowledge of the effect on its stability.

KEY-WORDS: FUNCTIONAL FOODS; FOOD - PROCESSING; FOOD STORAGE; PHENOLIC COMPOUNDS; Brassica.

\section{REFERÊNCIAS}

1 ACHINEWHU, S.C.; HART, A.D. Effect of processing and storage on the ascorbic acid (vitamin C) content of some pineapple varieties grown in the Rivers State of Nigeria. Plant Foods for Human Nutrition, Dordrecht, v. 46, n. 4, p. 335-337, 1994

2 ADA. American Dietetic Association. Position of the American Dietetic Association: functional food. Journal of American Dietetic Association, Philadelphia, v.104, n.5, p.814-826, 2004.

3 ARTS, I.C.W.; VAN DE PUTTE, B.; HOLLMAN, P.C.H. Catechin contents of foods commonly consumed in the Netherlands. 2. Tea, wine, fruit juices, and chocolate milk. Journal of Agricultural and Food Chemistry, Washington, v.48, p.1752-1757, 2000.

4 BALASUNDRAM, N.; SUNDRAM, K.; SAMMAN, S. Phenolic compounds in plants and agri-industrial by-products: antioxidant activity, occurrence, and potential uses. Food Chemistry, London, v. 99, p. 191-203, 2006. 
5 BARRETT, D.M.; WEAKLEY, C.; WATNIK, M. Qualitative and nutritional differences in processing tomatoes grown under commercial organic and conventional production systems. Journal of Food Science, Chicago, v.72, n. 9, p.C441-C451, 2007.

6 BATTAGLIA, A.M. O setor produtivo e funcionais. In: I CONGRESSO INTERNACIONAL SOBRE ALIMENTOS FUNCIONAIS - CIÊNCIA, INOVAÇÃO E REGULAMENTAÇÃO, 2006, São Paulo. Palestra... São Paulo: FIESP, 2006.

7 BEVERIDGE, T. Haze and cloud in apple juices. CRC - Critical Reviews in Food Science and Nutrition, Boca Raton, v.37, p.75-91, 1997.

8 BOVY, A.; DE VOS, R.; KEMPER, M.; SCHIJLEN, E.; PERTEJO, M.A.; MUIR, S. et al. High-flavonol tomatoes resulting from the heterologous expression of the maize transcription factor genes Ic and c1. The Plant Cell, Waterbury, v.14, p.2509-2526, 2002.

9 BRECHT, J.K.; RITENOUR, M.A.; HAARD, N.F.; CHISM, G.W. Postharvest physiology of edible plant tissues. In: DAMORADAN, S.; PARKIN, K.L.; FENNEMA, O.R. Fennema's food chemistry. $4^{\text {th }}$ ed. Boca Ranton: CRC Press, 2008. p.1042-1043.

10 BURDOCK, G.A.; CARABIN, I.G.; GRIFFTHS, J.C. The importance of GRAS to the functional food and nutraceutical industries. Toxicology, London, v. 221, p. 17-27, 2006.

11 CARVALHO, R. A.; FERREIRA, C.A.P.; NASCIMENTO JUNIOR, J.D.B.; MENEZES, A.J.E.A.; SUZUKE, E.; SASAKI, G. Análise econômica da produção de acerola no município de Tomé-Açu, Pará. Belém: Embrapa Amazônia Oriental, 2000. 21 p. (Embrapa Amazônia Oriental. Documentos, 49).

12 CHANG, C.H.; LIN, H.Y.; CHANG, C.Y.; LIU, Y.C. Comparisons on the antioxidant properties of fresh, freeze-dried and hot air dried tomatoes. Journal of Food Engineering, New York, v. 77, p. 478-485, 2006.

13 DAXENBICHLER, M.E.; SPENCER, G.F.; CARLSON, D.G.; ROSE, G.B.; BRINKER, A.M.; POWELL, R.G. Glucosinolate composition of seeds from 297 species of wild plants. Phytochemistry, New York, v.30, n.8, p.2623-2638, 1991.

14 DEKKER, M.; VERKERK, R.; VAN DER SLUIS, A.A.; KHOKHAR, S.; JONGEN, W.M.F. Analysing the antioxidant activity of food products: processing and matrix effects. Toxicology in Vitro, London, v.13, p. 797-799, 1999.

15 FINLEY, J.W. Proposed criteria for assessing the efficacy of cancer reduction by plant foods enriched in carotenoids, glucosinolates, polyphenols and selenocompounds. Annals of Botany, London, v.95, p.1075-1096, 2005.

16 GAHLER, S.; OTTO, K.; BOHM, V. Alterations of vitamin C, total phenolics, and antioxidant capacity as affected by processing tomatoes to different products. Journal of Agricultural and Food Chemistry, Washington, v.51,n.27, p. 7962-7968, 2003.

17 GatTUSO, G.; BARRECA, D.; GARGIULLI, C.; LEUZZI, U.; CARISTI, C. Flavonoid composition of Citrus juices. Molecules, Basel, v. 12, p. 1641-1673, 2007.

18 GERARD, K.A.; ROBERTS, J.S. Microwave heating of apple mash to improve juice yield and quality. LebensmittleWissenschaft Und Technologie, London, v.37, p.551-557, 2004.

19 GIL-IZQUIERDO, A.; GIL, M.I.; FERRERES, F. Effect of processing techniques at industrial scale on orange juice antioxidant and beneficial health compounds. Journal of Agricultural and Food Chemistry, Washington, v.50, n. 18, p.5107-5114, 2002.

20 GIMENEZ, R.; CABRERA, C.; OLALLA, M.; RUIZ, M.D.; LÓPEZ, M.C. Ascorbic acid in diet supplements: loss in the manufacturing process and storage. International Journal of Food Sciences and Nutrition, London, v. 53, n. 6 , p. 509-518, 2002

21 GOODRICH, R.M.; ANDERSON, J.L.; STOEWSAND, G.S. Glucosinolate changes in blanched broccoli and brussels sprouts. Journal of Food Processing and Preservation, San Francisco, v.13, n.4, p.275-280, 1989.

22 GRIZARD, D.; DALLE, M.; BARTHOMEUF, C. Changes in insulin and corticosterone levels may partly mediate the hypolipidemic effect of guar gum and low-molecular weight pectin in rats. Nutrition Research, New York, v. 21, p. 1185-1190, 2001.

23 HAKKINEN, S.H.; KARENLAMPI, S.O.; MYKKANEN, H.M; TORRONEN, A.R. Influence of domestic processing and storage on flavonol contents in berries. Journal of Agricultural and Food Chemistry, Washington, v. 48, p. 29602965, 2000.

24 HALVORSEN, B. L.; HOLTE, K.; MYHRSTAD, M. C. W.; BARIKMO, I.; HVATTUM, E.; FAGERTUN REMBERG, S.; WOLD, A.-B.; HAFFNER, K.; BAUGEROD, H.; FROST ANDERSEN, L.; MOSKAUG, J. O.; JACOBS, D. R.; BLOMHOFF, R. A systematic screening of total antioxidants in dietary plants. The Journal of Nutrition, Bethesda, v.132, p.461-471, 2002. 
26 IZZO, M.; NINESS, K. Formulating nutrition bars with inulin and oligofructose. Cereal Foods World, St. Paul, v. 46, n.3, p. 102-106, 2001.

27 JACKSON, S.J.T.; SINGLETARY, K.W. Sulforaphane inhibits human MCF-7 mammary cancer cell mitotic progression and tubulin polymerization. The Journal of Nutrition, Bethesda, v.134, p.2229-2236, 2004.

28 KELLY, P.J.; BONES, A.; ROSSITER, J.T. Sub-cellular immunolocalization of the glucosinolate sinigrin in seedlings of Brassica juncea. Planta Medica, Stuttgart, v.206, p. 370-377, 1998.

29 KLOPOTEK, Y.; OTTO, K.; BOHM, V. Processing strawberries to different products alters contents of vitamin C, total phenolics, total anthocyanins, and antioxidant capacity. Journal of Agricultural and Food Chemistry, Washington, v. 53, n.14, p.5640-5646, 2005.

30 KRISTAL, A.R.; LAMPE, J.W. Brassica vegetables and prostate cancer risk: a review of the epidemiological evidence. Nutrition and Cancer, New York, v.42, p.1-9, 2002

31 LANDBO, A.K.R.; PINELO, M.; VIKBJERG, A.F.; LET, M.B.; MEYER, A.S. Protease-assisted clarification of black currant juice: synergy with other clarifying agents and effects on the phenol content. Journal of Agricultural and Food Chemistry, Washington, v.54, n.18, p.6554-6563, 2006.

32 LESTER, G.E.; MANTHEY, J.A.; BUSLIG, B.S. Organic vs conventionally grown rio red whole grapefruit and juice: comparison of production inputs, market quality, consumer acceptance, and human health bioactive compounds. Journal of Agricultural and Food Chemistry, Washington, v.55, n.11, p.4474-4480, 2007.

33 LOMBARDI-BOCCIA, G.; LUCARINI, M.; LANZI, S.; AGUZZI, A.; CAPPELLONI, M. Nutrients and antioxidant molecules in yellow plums (Prunus domestica L.) from conventional and organic productions: a comparative study. Journal of Agricultural and Food Chemistry, Washington, v.52, p.90-94, 2004.

34 MAIA, G.A.; SOUSA, P.H.M.; SANTOS, G.M.; SILVA, D.S.; FERNANDES, A.G.; PRADO, G.M. Effect of the processing on some components of acerola juice. Ciência e Tecnologia de Alimentos, Campinas, v. 27, n. 1, p. 130-134, 2007.

35 MANACH, C.; SCALBERT, A.; MORAND, C.; REMESY, C.; JIMENEZ L. Polyphenols: food sources and bioavaiability. The American Journal of Clinical Nutrition, Bethesda, v.79, p.727-747, 2004.

36 MATSUURA, F. C. A. U.; FOLEGATTI, M. L. S.; FERREIRA, D. C.; CARDOSO, R.L. Produção de geléia mista de maracujá e acerola com alto teor de vitamina C. In: CONGRESSO BRASILEIRO DE FRUTICULTURA, 17., 2002, Belém. Anais... Belém: Sociedade Brasileira de Fruticultura, 2002. 1 CD-ROM.

37 MITHEN, R.F.; DEKKER, M.; VERKERK, R.; RABOT, S.; JOHNSON, I.T. The nutritional significance, biosynthesis and bioavailability of glucosinolates in human foods. Journal of the Science of Food and Agriculture, London, v.80, n.7, p.967-984, 2000.

38 MORENO, D.A.; CARVAJAL, M.; LÓPEZ-BERENGUER, C.; GARCÍA-VIGUERA, C. Chemical and biological characterisation of nutraceutical compounds of broccoli. Journal of Pharmaceutical and Biomedical Analysis, London, V. 41, p. 1508-1522, 2006.

39 MOTTA, M. Panorama do mercado de funcionais. In: CONGRESSO INTERNACIONAL SOBREALIMENTOS FUNCIONAIS - CIÊNCIA, INOVAÇÃO E REGULAMENTAÇÃO, 2006, São Paulo. Palestra... São Paulo: FIESP, 2006.

40 NAMIKI, M. Antioxidants/antimutagens in food. CRC - Critical Reviews in Food Science and Nutrition, Boca Raton, v.29, p.273-300, 1990.

41 NICOLI, M.C.; ANESE, M.; PARPINEL, M. Influence of processing on the antioxidant properties of fruit and vegetables. Trends in Food Science and Technology, New York, v. 10, n. 3, p. 94-100, 1999.

42 ODRIOZOLA-SERRANO, I.; SOLIVA-FORTUNY, R.; MARTIN-BELLOSO, O. Effect of minimal processing on bioactive compounds and color attributes of fresh-cut tomatoes. Lebensmittle-Wissenschaft Und Technologie, London, v. 41 , p. $217-226,2008$.

43 PERERA, C. O.; BALDWIN, E. A. Biochemistry of fruits and its implications on processing. In: ARTNEY, D.; ASHURT P. R. (Eds.). Fruit processing: nutrition, product, quality management. $2^{\text {nd }}$ ed. New York: AN ASPEN Publication, 2001. p. 26-27.

44 ROBBINS, R.J. Phenolic acids in foods: an overview of analytical methodology. Journal of Agricultural and Food Chemistry, Washington, v. 51, n.10, p.2866-2887, 2003.

45 SAHLIN, E.; SAVAGE, G.P.; LISTER, C.E. Investigation of the antioxidant properties of tomatoes after processing. Journal of Food Composition and Analysis, New York, v. 17, p. 635-647, 2004.

46 SCHIEBER, A.; STINTZING, F.C.; CARLE, R. By-products of plant food processing as a source of functional compounds - recent developments. Trends in Food Science and Technology, New York, v.12, p.401-413, 2001. 
47 SEMENSATO, L.R. Caracterização físico-química de frutos genótipos de acerola (Malpighia sp.), cultivados em Anápolis-GO, processamento e estabilidade de seus produtos. Goiânia, 1997. 74 p. Dissertação (Mestrado em Agronominas), Universidade Federal de Goiânia (UFG).

48 SHAPIRO, T.A.; FAHEY, J.W.; WADE, K.L.; STEPHENSON, K.K.; TALALAY, P. Chemopreventive glucosinolates and isothiocyanates of broccoli sprouts: metabolism and excretion in humans. Cancer Epidemiology, Biomarkers \& Prevention, New Milford, v.10, p.501-508, 2001.

49 SIEBERT, K.J. Effects of protein-polyphenol interactions on beverage haze, stabilization, and analysis. Journal of Agricultural and Food Chemistry, Washington, v.47, p.353-363, 1999.

50 SIEBERT, K.J.; CARRASCO, A.; LYNN, P. Y. Formation of proteinpolyphenol haze in beverages. Journal of Agricultural and Food Chemistry, Washington, v.44, p.1997-2005, 1996.

51 SIEBERT, K.J.; TROUKHANOVA, N.V.; LYNN, P.Y. Formation of protein-polyphenol haze in beverages. Journal of Agricultural and Food Chemistry, Washington, v.44, p.80-85, 1996.

52 SONG, L.; THORNALLEY, P.J. Effect of storage, processing and cooking on glucosinolate content of Brassica vegetables. Food and Chemical Toxicology, London, v.45, p. 216-224, 2007.

53 SOUTO, A. Supermolécula pode prevenir doenças. PUCRS- Informação, Porto Alegre, v. 30, n.133, p.6-9, 2007.

54 STAHL, W.; SIES, H. Uptake of lycopene and its geometrical isomers is greater from heat processed than from unprocessed tomato juice in humans. The Journal of Nutrition, Bethesda, v.122, n.11, p. 2161-2166, 1992.

55 STRINGHETA, P.C.; VILELA, M.A.P.; OLIVEIRA, T.T.; NAGEM, T.J. Alimentos "funcionais": conceitos, contextualização e regulamentação. Juiz de Fora: Templo, 2007. 246 p.

56 SWADLING, I. The regulation and marketing of functional foods worldwide. Food Info Online. Disponível em: <http:/ /www.foodsciencecentral.com/fsc/ixid3732>. Acesso em: 23 janeiro 2009.

57 TOOR, R.K.; SAVAGE, G.P. Changes in major antioxidant components of tomatoes during post-harvest storage. Food Chemistry, London, v. 99, p.724-727, 2006a

58 TOOR, R.K.; SAVAGE, G.P. Effect of semi-drying on the antioxidant components of tomatoes. Food Chemistry, London, v. 94, p. 90-97, 2006b.

59 VAN DER SLUIS, A.A.; DEKKER, M.; SKREDE, G.; JONGEN, W. M. F. Activity and concentration of polyphenolic antioxidants in apple juice. 1. Effect of existing production methods. Journal of Agricultural and Food Chemistry, Washington, v.50, p.7211-7219, 2002

60 VAN DER SLUIS, A. A.; DEKKER, M.; VAN BOEKEL, M.A.J.S. Activity and concentration of polyphenolic antioxidants in apple juice. 3. Stability during storage. Journal of Agricultural and Food Chemistry, Washington, v.53, p.10731080, 2005.

61 VOLDEN, J.; BORGE, G.I.A.; BENGTSSON, G.B.; HANSEN, M.; THYGESEN, I.E.; WICKLUND, T. Effect of thermal treatment on glucosinolates and antioxidant-related parameters in red cabbage (Brassica oleracea L. ssp. capitata f. rubra). Food Chemistry, London, v. 109, p. 595-605, 2008.

62 WATHELET, J.-P.; MABON, N.; FOUCART, M.; MARLIER, M. Influence of blanching on the quality of Brussels sprouts (Brassica oleracea L. cv. gemmifera). Sciences des Aliments, Cachan, v.16, n.4, p.393-402, 1996.

63 WENNBERG, M.; EKVALL, J.; OLSSON, K.; NYMAN, M. Changes in carbohydrate and glucosinolate composition in white cabbage (Brassica oleracea var. capitata) during blanching and treatment with acetic acid. Food Chemistry, London, v.95, n.2, p.226-236, 2006.

64 XIANQUAN, S.; SHI, J.; KAKUDA, Y.; YUEMING, J. Stability of lycopene during food processing and storage. Journal of Medicinal Food, New Rochelle, v.8, n.4, p.413-422, 2005.

65 YAMASHITA, F.; BENASSI, M. T.; TONZAR, A. C.; MORIYA, S.; FERNANDES, J. G. Produtos de acerola: estudo da estabilidade de vitamina C. Ciência e Tecnologia de Alimentos, Campinas, v. 23, n. 1, p. 92-4, 2003.

66 ZHANG, D. L.; HAMAUZU, Y. Phenolics, ascorbic acid, carotenoids and antioxidant activity of broccoli and their changes during conventional and microwave cooking. Food Chemistry, London, v.88, n.4,p. 503-509, 2004. 